\title{
A FÓRMULA CROMÁTICA NO TESTE DAS PIRÂMIDES COLORIDAS DE PFISTER EM DIFERENTES FAIXAS ETÁRIAS
}

THE CHROMATIC FORMULA IN PFISTER'S COLORED PYRAMIDS TEST AMONG DIFFERENT AGE GROUPS

\section{LA FÓRMULA CROMÁTICA EN EL TEST DE LAS PIRÁMIDES COLORIDAS DE PFISTER EN DIFERENTES GRUPOS DE EDAD}

\author{
Anna Elisa Villemor Amaral \\ Fabiola Cristina Biasi** \\ Pâmela Malio Pardini Pavan ${ }^{* * *}$ \\ Raquel Rossi Tavella ${ }^{* * * *}$ \\ Lucila Moraes Cardoso ${ }^{* * * * *}$
}

\begin{abstract}
RESUMO
O teste das pirâmides coloridas de Pfister (TPC) é uma técnica que auxilia na compreensão de aspectos emocionais e cognitivos. $\mathrm{O}$ indicador fórmula cromática do TPC reflete a maturidade emocional e, por isso, objetivou-se identificar evidências de validade desse indicador comparandose o desempenho de pessoas em diferentes faixas etárias. Para tal, foram comparados os protocolos de 39 crianças de 6 anos, 47 adolescentes de 12 anos e 73 estudantes universitários. Observou-se que a frequência de crianças que apresentaram fórmulas amplas foi significativamente maior que dos demais grupos $\left(x^{2}=23,418 ; p<0,001\right)$ e que a amplitude moderada foi mais frequente no grupo de universitários $\left(x^{2}=17,712 ; p<0,001\right)$. Em relação à estabilidade nas escolhas, notou-se que a fórmula cromática estável foi mais frequente nas crianças $\left(x^{2}=17,152 ; p=0,001\right)$ e que a instável foi significativamente mais frequente nos universitários $\left(x^{2}=11,652 ; p=0,001\right)$. Concluiu-se que o TPC contribui para diferenciar níveis de maturidade emocional de acordo com as faixas etárias.
\end{abstract}

Texto recebido em 15 de julho de 2013 e aprovado para publicação em 8 de setembro de 2014.

Doutora em Ciências pela Unifesp/EPM; docente do Programa de Pós-Graduação Stricto Sensu em Psicologia da Universidade São Francisco. Endereço: Rua Alexandre Barbosa Rodrigues, 45 - Centro, Itatiba-SP, Brasil. CEP: 13251-900.

E-mail: anna.villemor@saofrancisco.edu.br.

** Mestra em Psicologia pela Universidade São Francisco; docente do Curso de Psicologia da Universidade São Francisco. Endereço: Rua Alexandre Barbosa Rodrigues, 45 - Centro, Itatiba-SP, Brasil. CEP: 13251-900.

E-mail: fabiola.biasi@usf.edu.br.

*** Mestra em Psicologia pela Universidade São Francisco. Endereço: Rua Alexandre Barbosa Rodrigues, 45 - Centro, Itatiba-SP, Brasil. CEP: 13251-900. E-mail: papavam@gmail.com

**** Mestra em Psicologia pela Universidade São Francisco; docente do curso de Psicologia no Centro Universitário Nossa Senhora do Patrocínio (Ceunsp). Endereço: Centro Universitário Nossa Senhora do Patrocínio, Faculdade Nossa Senhora do Patrocínio - Centro, Itu-SP, Brasil. CEP: 13300-903. Telefone: (11) 4013-9935. E-mail: raqueltavella@yahoo.com.br.

****** Doutora em Psicologia pela Universidade São Francisco; docente do Programa de Graduação e Pós-Graduação em Educação da Universidade Estadual do Ceará (UECE). Endereço: Universidade Estadual do Ceará, Centro de Humanidades, Fundação Universidade Estadual do Ceará - UECE - Itaperi, Fortaleza-CE, Brasil. CEP: 60714-903.

E-mail: lucilamcardoso@yahoo.com.br. 
Palavras-chave: Avaliação psicológica. Evidências de validade. Teste das pirâmides coloridas.

\section{ABSTRACT}

The Pfister's test is an expressive technique of psychological assessment that helps the understanding of the emotional and cognitive aspects. The chromatic formula reflects the emotional maturity so this work aimed to identify evidence of the validity of this indicator by comparing the results of people in different age groups. In order to do this, the protocols of 39 children of 6 year olds, 47 participants of 12 year olds and 73 college students were compared. It was observed that the frequency of children with a broad formula was significantly higher than the other groups $\left(x^{2}=23.418\right.$; $p<0.001)$ and moderate amplitude was more frequent in the college group $\left(x^{2}=17.712 ; p<0.001\right)$. With concerns about stability in the choices, it was noted that the stable formula was more frequent in children $\left(x^{2}=17.152\right.$; $p=0.001)$ and the unstable was significantly more frequent among the college students $\left(x^{2}=11.652 ; p=0.001\right)$. These results concludes that the Pfister contributes to differentiate emotional maturity levels according to age groups.

Keywords: Psychological Assessment. Evidence of validity. Pfister's test.

\section{RESUMEN}

El test de las pirámides coloridas de Pfister (TPC) es una técnica que contribuye a la comprensión de los aspectos emocionales y cognitivos. El indicador fórmula cromática del TPC refleja la madurez emocional y, por lo tanto, se procuró identificar evidencias de validez de este indicador comparando el desempeño de las personas en diferentes grupos de edad. Para ello, fueron comparados los protocolos de 39 niños de 6 años, 47 adolescentes de 12 años y 73 estudiantes universitarios. Se observó que la frecuencia de los niños con fórmulas amplias fue significativamente más alta que los otros grupos $\left(x^{2}=23,418 ; p<0,001\right)$ y la amplitud moderada fue más frecuente en el grupo de los estudiantes universitarios $\left(x^{2}=17,712 ; p<0,001\right)$. Cuanto a la estabilidad en las elecciones, se señaló que la fórmula cromática estable fue más frecuente en los niños $\left(x^{2}=17,152 ; p=0,001\right)$ y la inestable fue significativamente más frecuente en los estudiantes universitarios $\left(x^{2}=11,652 ; p=0,001\right)$. Con esto se pudo demonstrar que el TPC contribuye a diferenciar los niveles de madurez emocional de acuerdo a las edades.

Palabras clave: Evaluación psicológica. Evidencias de validez. El test de las pirámides coloridas. 


\section{INTRODUÇÃO}

$\mathrm{N}$ a última década, a área da Avaliação Psicológica tem sido marcada pelos avanços científicos e pela consolidação dessa prática como um importante campo de atuação para a Psicologia brasileira (Primi, 2010). Os avanços são notórios tanto do ponto de vista quantitativo quanto qualitativo, observando-se um rigor maior no que se refere às qualidades psicométricas dos instrumentos de avaliação psicológica.

Entretanto, ainda que se notem avanços, uma rápida busca no Sistema de Avaliação dos Testes Psicológicos (SATEPSI) (Conselho Federal de Psicologia [CFP], 2003), revela uma escassa quantidade de instrumentos que possam ser utilizados para avaliação de aspectos da dinâmica de personalidade de crianças ou adolescentes e que tenham um parecer favorável para uso. Numa consulta realizada ao Satepsi, em setembro de 2014, os únicos quatro instrumentos que atendiam a essa demanda e que estavam com o parecer favorável foram o Rorschach, o HTP (teste da árvore, casa e pessoa), o teste de apercepção infantil - figuras de animais (CAT-A) e o teste das pirâmides coloridas de Pfister.

A escassez de instrumentos de avaliação psicológica específicos para crianças se torna um desafio, pois uma avaliação correta e pautada em critérios científicos é fundamental para garantir intervenções mais eficazes nas situações em que existam queixas de dificuldades de adaptação ou outros problemas. Os primeiros anos de vida são os formadores da personalidade, ao mesmo tempo em que se trata de um período no qual os sujeitos estão mais vulneráveis a alterações sociais e fisiológicas (Pérez-Ramos, 2000), demandando intervenções sempre que se observam problemas que comprometam o pleno e saudável desenvolvimento.

Diante da necessidade de instrumentos para avaliação de crianças e das características lúdicas do teste das pirâmides coloridas de Pfister (TPC), estão sendo realizados diversos estudos que buscam estabelecer normas do TPC para crianças. Uma revisão da literatura revelou que, nos últimos dez anos, foram publicados 12 estudos com o TPC, dos quais 2 buscavam normas para adultos e 6 por evidências de validades da técnica, sendo que em um deles a amostra era de crianças (Silva \& Cardoso, 2012).

O TPC é uma técnica expressiva que propicia primordialmente a avaliação da dinâmica emocional, mas também de aspectos cognitivos da personalidade do indivíduo. Ele é usado há mais de 50 anos na clínica e em pesquisa, tendo boa aceitação entre os profissionais do campo da psicologia (Villemor-Amaral, 2012). A aceitação do método também é inferida pela existência de vários trabalhos relacionados às aplicações dessa técnica em diversos campos da Psicologia, principalmente nas áreas de clínica e saúde mental (Carnio \& Loureiro, 1993; Oliveira, Pasian \& Jacquemin, 2001). 
No que se refere à verificação de suas qualidades psicométricas, foram realizados estudos normativos com o TPC, tanto para 277 indivíduos adultos do estado de São Paulo (Villemor-Amaral, Primi, Farah, Cardoso \& Franco, 2003) quanto para 83 adultos de Estados do Nordeste brasileiro (Villemor-Amaral, Pianowski \& Gonçalves, 2008). Mais recentemente, foram publicados estudos normativos do TPC com idosos (Bastos-Formighieri \& Pasian, 2012), com crianças (Villemor-Amaral, Cardoso, Pavan, Tavella, Biasi \& Miguel, 2014b) e com adolescentes de idade entre 12 e 14 anos (Pasian, Barroso \& Theodozio, 2014).

Dos 4 estudos que se dedicaram à fidedignidade do TPC, 3 foram de precisão entre avaliadores, sendo 2 para o aspecto formal e fórmula cromática (VillemorAmaral, 2012; Villemor-Amaral, Cardoso, Pavan, Tavella \& Biasi, 2014a) e 1 para o aspecto formal (Farah, 2010). Nos três estudos, as concordâncias entre avaliadores variaram entre $86 \%$ e $88 \%$, sendo considerados bons índices de precisão. $\mathrm{O}$ outro estudo buscou precisão temporal, encontrando bons níveis de estabilidade para o aspecto formal $(r=0,80, p<0,001$ para tapetes, $r=0,48$, $\mathrm{p}=0,015$ para formações e $\mathrm{r}=0,30, \mathrm{p}=0,144$ para estruturas) e a fórmula cromática $(\mathrm{r}=19,91, \mathrm{p}=0$,001) (Villemor-Amaral, Miguel, Biasi, Migoranci, Pavan, \& Tavella, 2012).

No que se refere aos estudos de evidências de validade, foram feitas investigaçōes com adultos, conforme consta do manual (Villemor-Amaral, 2012), com idosos (Oliveira et al., 2001), com crianças (Resende \& Chagas, 2014; Villemor-Amaral, Pardini, Tavella, Biasi \& Migoranci, 2012), com crianças surdas (Cardoso \& Capitão, 2007) e com adolescentes que apresentaram sintomas depressivos no inventário Beck de depressão (BDI) (Adib, 2008). Todos esses estudos apresentaram evidências de validade para indicadores do TPC, contribuindo para reforçar as qualidades psicométricas do instrumento.

Além disso, há estudos que demonstram evidências de validade do TPC para diferenciar grupos normativos de psicopatológicos, conferindo-lhe caráter complementar no psicodiagnóstico (Villemor-Amaral, Farah \& Primi, 2004; Villemor-Amaral, Primi, Farah, Silva, Cardoso \& Franco, 2004; Villemor-Amaral, Primi, Franco, Farah, Cardoso \& Silva, 2005; Villemor-Amaral, Silva \& Primi, 2002; Villemor-Amaral, Silva \& Primi, 2003). Esses estudos de normatização, de precisão e de evidência de validade foram justificados pela importância de qualificar os alcances e limites no uso do TPC. Ainda assim, destaca-se que nenhum dos estudos se ateve aos indicadores da fórmula cromática do TPC.

A fórmula cromática considera a incidência das cores na sequência das três pirâmides, ou seja, nesse indicador, é analisado em que medida a pessoa utiliza 
a variedade de cores que o teste apresenta e o quanto ela é constante nessas escolhas no decorrer da primeira para a terceira pirâmide. Essa fórmula compõese de quatro algarismos que, quando combinados, representam a amplitude e a constância das escolhas.

$\mathrm{O}$ primeiro algarismo se refere à quantidade de cores que foram utilizadas nas três pirâmides, denominado "algarismo de constância absoluta" (CA), o segundo indica quantas cores foram empregadas em duas das três pirâmides, não importando em quais, e é chamado "algarismo de constância relativa" (CR), o terceiro representa a quantidade de cores usada em apenas uma das pirâmides chamado "variabilidade" (V) e o último é o "algarismo de ausência" (AUS), que indica a quantidade de cores omitidas ou rejeitadas, ou seja, não utilizadas pela pessoa nas suas pirâmides (CA: CR: V: AUS).

A amplitude nas escolhas é atribuída por meio da análise do algarismo AUS, sendo que valores 0 ou 1 indicam uma fórmula ampla, valores entre 2 e 4 apontam para uma amplitude moderada e, por fim, valores iguais ou superiores a 5 caracterizam uma fórmula restrita. Desse modo, valores diminuídos no algarismo AUS indicam que a pessoa utilizou muitas das cores disponíveis, sugerindo elevada abertura para os estímulos e às experiências. Seguindo o mesmo raciocínio, observa-se que o índice elevado no algarismo de ausência ocorre quando o examinando optou por usar poucas cores, demonstrando menor receptividade aos estímulos.

A constância nas escolhas pode ser verificada por meio da diferença numérica entre os três primeiros algarismos. Desse modo, valores aumentados na direção do algarismo CA indicam estabilidade, valores aumentados na direção $\mathrm{V}$ sugerem instabilidade e valores mais elevados em CR denotam flexibilidade. Essa variável indica em que medida a pessoa tende a se manter constante em suas escolhas ou se está mais propensa a variá-las ao longo do teste. A combinação da amplitude e constância resulta em nove tipos de fórmulas:
a) ampla e estável;
b) ampla e flexível;
c) ampla e instável;
d) moderada e estável;
e) moderada e flexível;
f) moderada e instável; 

g) restrita e estável;
h) restrita e flexível; e, finalmente,
i) restrita e instável.

Pesquisas relacionadas à fórmula cromática sugerem que o número de cores utilizadas é uma característica que depende da idade. A amplitude cromática considerada ampla é típica da infância e tende a diminuir com o tempo, conforme já apontaram Heiss e Halder (1983). No que se refere à estabilidade nas escolhas, esses mesmos autores mencionaram a importância de esse indicador ser associado a outras características de instabilidade e que é preciso muita cautela ao tentar interpretá-lo de modo isolado. Para buscar interpretaçōes sobre o significado desses indicadores, foram considerados alguns postulados das teorias do desenvolvimento infantil.

Diversos teóricos discutem sobre os processos cognitivos e emocionais típicos de diferentes períodos do desenvolvimento, como exemplo Piaget, Wallon e Erikson. Piaget dedicou-se a compreender os processos do pensamento. Para ele, as pessoas herdam esquemas, isto é, estruturas biológicas que permitem o surgimento de estruturas mentais. Essas estruturas possibilitam o desenvolvimento em períodos relativamente comuns para todas as crianças, assim o desenvolvimento infantil seria marcado por diferentes etapas que ocorrem de modo relativamente universal (Cole \& Cole, 2003).

Segundo Dantas (1992), na visão de Henri Wallon, a afetividade é uma das dimensões do sujeito, correspondendo a uma fase mais arcaica do desenvolvimento. Para ele, o desenvolvimento é como uma construção progressiva em que as fases ocorrem alternando a predominância da esfera afetiva e da cognitiva. Tanto para Piaget quanto para Wallon, aos 6 anos de idade, há uma transição das etapas do desenvolvimento, começando a haver um predomínio do funcionamento cognitivo. Desse modo, há um interesse por parte das crianças para o conhecimento e conquista do mundo exterior.

$\mathrm{Na}$ puberdade, a crise causada pelas mudanças, inclusive corporais, que ocorrem nesse período faz surgir a necessidade de uma nova definição da personalidade, colocando em evidência as questóes pessoais, morais e existenciais. Nesse período, há a retomada do predomínio da afetividade (Dantas, 1992).

Para Erikson (1976), a dimensão psicológica dos adolescentes é intrínseca à história de vida e às relações socioculturais dos jovens. Sabe-se que essas interações sociais influenciam diretamente na dinâmica emocional e no funcionamento cognitivo dos jovens. Ainda para Erikson, é desejável que esse processo de 
adolescer resulte em indivíduos saudáveis, que apresentem certa unidade de personalidade e consigam lidar com o mundo e consigo mesmos. Por outro lado, muitas vezes, o início da fase adulta é marcado pelo ingresso no ensino superior, o que, de acordo com Santos e Suehiro (2007), pode ser potencializador de crises no desenvolvimento dos jovens nesse período.

De modo sintético, pode-se dizer que o desenvolvimento humano é marcado por eventos que ocorrem em fases distintas da vida e promovem algumas dinâmicas emocionais comuns a cada etapa. Partindo-se do pressuposto de que o TPC é uma técnica que avalia aspectos da dinâmica emocional e estruturação cognitiva, acredita-se que ele pode diferenciar alguns dos aspectos típicos do desenvolvimento humano. Tem-se, portanto, a hipótese de que as crianças apresentem maior amplitude cromática, pois estariam mais receptivas aos diversos estímulos que recebem, enquanto os adolescentes e adultos, por já terem características de personalidade mais constantes, apresentariam escolhas mais estáveis. Desse modo, este trabalho tem o objetivo de comparar as fórmulas cromáticas apresentadas nas diferentes faixas etárias e verificar se esse indicador consegue diferenciar aspectos da dinâmica emocional característica em cada uma dessas fases do desenvolvimento humano.

\section{MÉTODO}

\subsection{Participantes/banco de dados}

As informações dos participantes foram obtidas em bancos de dados de amostras normativas constantes dos manuais do TPC para crianças (VillemorAmaral, 2014) e adultos (Villemor-Amaral, 2012). O banco de dados foi composto por participantes selecionados por meio de amostra não probabilística de conveniência, que preenchiam como critério de inclusão a ausência de histórico de busca de ajuda psiquiátrica ou psicológica no caso de adultos e, para as crianças e jovens, não ter queixas específicas na escola e estarem frequentando a série compatível com a idade. Todos os aplicadores eram psicólogos ou estudantes de Psicologia que receberam um treinamento prévio pela coordenadora da pesquisa, tanto no que se refere à administração e codificação dos instrumentos quanto para organização do banco de dados, que requisitou o uso de programas específicos criados especialmente para fins de pesquisa. Os aplicadores receberam instruções estritas sobre como estabelecer um rapport, como aplicar, responder a questôes e fizeram várias aplicaçôes treino prévias à coleta de dados.

O banco contava com 721 protocolos de participantes de ambos os sexos e diversas faixas etárias. Do banco de dados selecionou-se 86 protocolos, sendo 
39 protocolos de crianças de 6 anos (24,1\%), 47 de jovens de 12 anos (29,7\%), de ambos os gêneros, de escolas públicas do interior do Estado de São Paulo, correspondendo à totalidade de indivíduos dessas faixas etárias. Selecionou-se também 76 adultos que correspondiam ao total de estudantes universitários (46,2\%), com idades variando de 18 a 29 anos e média de 22 anos, também de ambos os gêneros e de diversos cursos, como Medicina, Engenharia e Psicologia, de uma instituição privada do interior do Estado de São Paulo.

\subsection{Instrumentos}

O Teste das Pirâmides Coloridas de Pfister (TPC) foi criado por Max Pfister (1888-1958) em 1951. O TPC consiste na execução de três pirâmides coloridas, conforme a preferência do examinando. $\mathrm{O}$ material necessário para a aplicação é composto de um jogo de três cartôes em papel pardo, no qual há uma pirâmide desenhada, formada de cinco degraus de quadrados de $2,5 \mathrm{~cm}$ de cada lado. A base é composta de 5 quadrados, as linhas seguintes 4 e 3 e, para terminar, 2 e 1 no vértice. $\mathrm{O}$ jogo de quadrículos coloridos compóe-se de dez cores, subdivididas em 24 tonalidades. Esse jogo deve conter no mínimo 45 quadrículos de cada tom para que o indivíduo tenha a possibilidade de construir todas as pirâmides com uma só tonalidade, caso assim o deseje. As cores que compõem o material do teste são 4 tonalidades de azul, verde e vermelho, 3 tonalidades de violeta, 2 tonalidades de amarelo, laranja e marrom e as cores preto, branco e cinza (Villemor-Amaral, 2012). As instruções para as aplicações do TPC foram pautadas no manual da técnica (Villemor-Amaral, 2012).

\subsection{Procedimentos}

Após a seleção e extração dos protocolos dos participantes de 6 anos, 12 anos e estudantes universitários com mais de 18 anos, esses protocolos foram armazenados numa nova planilha, e as análises estatísticas foram feitas utilizandose o programa SPPS, versão 20. Neste trabalho, somente serão descritos os resultados referentes à fórmula cromática. Como a fórmula cromática é uma variável de nível e mensuração nominal, optou-se por utilizar o qui-quadrado para fazer a comparação da frequência das variáveis entre os três grupos estudados, utilizando-se nível de significância de $p<0,05$. Além disso, destaca-se que, como os grupos tinham diferenças no tamanho da amostra, optou-se por utilizar post hoc Kruskal Wallis Test, que faz comparação de média entre três ou mais grupos quando a distribuição da amostra não é normal (igual) (Dancey \& Reidy, 2006). 


\section{RESULTADOS}

Primeiramente foram feitos procedimentos de análise estatística descritiva e a comparação da frequência das nove categorias de fórmula cromática entre os três grupos, não havendo resultados estatisticamente significativos. Por essa razão, optou-se por fazer análises separando-se os dois fatores que compõem a fórmula cromática, ou seja, a amplitude (tabela 1) e a estabilidade (tabela 2).

Tabela 1 - Comparação da amplitude cromática do teste de Pfister entre as diferentes idades

\begin{tabular}{|c|c|c|c|c|c|c|c|c|}
\hline \multirow{2}{*}{$\begin{array}{c}\text { Amplitude } \\
\text { cromática }\end{array}$} & \multicolumn{2}{|c|}{$\mathbf{6}$ anos (n=39) } & \multicolumn{2}{|c|}{$\mathbf{1 2}$ anos (n=47) } & \multicolumn{2}{|c|}{$\begin{array}{c}\text { 18 anos ou } \\
\text { mais (n=73) }\end{array}$} & \multirow{2}{*}{$\mathbf{X}^{\mathbf{2}}$} & \multirow{2}{*}{$\boldsymbol{p}$} \\
\cline { 2 - 8 } & $\mathbf{f}$ & $\mathbf{\%}$ & $\mathbf{F}$ & $\mathbf{\%}$ & $\mathbf{f}$ & $\mathbf{\%}$ & & \\
\hline Ampla & 36 & 92,3 & 32 & 68,1 & 34 & 46,6 & 23,418 & $<\mathbf{0 , 0 0 1}$ \\
\hline Moderada & 2 & 5,1 & 10 & 21,3 & 30 & 41,1 & 17,712 & $<\mathbf{0 , 0 0 1}$ \\
\hline Restrita & 1 & 2,6 & 5 & 10,6 & 9 & 12,3 & 2,931 & 0,231 \\
\hline Total & 39 & 100 & 47 & 100 & 73 & 100 & & \\
\hline
\end{tabular}

Fonte: elaborado pelas autoras.

Os resultados relativos à amplitude das fórmulas cromáticas apresentados na tabela 1 revelaram diferenças estatisticamente significativas entre os grupos. Nos três grupos, houve predomínio da fórmula ampla, caracterizada pela escolha de grande quantidade de cores, mostrando menor seletividade e maior receptividade de estimulação cromática. Já a fórmula restrita se apresentou reduzida nos três conjuntos de indivíduos avaliados.

A partir das análises post hoc, nota-se que a fórmula cromática ampla foi significativamente mais frequente no grupo de crianças, enquanto que a fórmula moderada foi significativamente mais frequente no grupo de universitários. Não houve diferenças significativas entre os grupos em relação à fórmula restrita.

Tabela 2 - Comparação da estabilidade cromática do teste de Pfister entre as diferentes idades

\begin{tabular}{|c|c|c|c|c|c|c|c|c|}
\hline \multirow{2}{*}{$\begin{array}{l}\text { Estabilidade } \\
\text { cromática }\end{array}$} & \multicolumn{2}{|c|}{$\begin{array}{l}6 \text { anos } \\
(n=39)\end{array}$} & \multicolumn{2}{|c|}{$\begin{array}{c}12 \text { anos } \\
(n=47)\end{array}$} & \multicolumn{2}{|c|}{$\begin{array}{l}18 \text { anos ou } \\
\text { mais }(n=73)\end{array}$} & \multirow[t]{2}{*}{$\mathrm{X}^{2}$} & \multirow[t]{2}{*}{$p$} \\
\hline & $f$ & $\%$ & $\mathbf{F}$ & $\%$ & $f$ & $\%$ & & \\
\hline Estável & 31 & 79,5 & 25 & 53,2 & 28 & 38,4 & 17,152 & $<0,001$ \\
\hline Flexível & 5 & 12,8 & 13 & 27,7 & 19 & 26 & 3,183 & 0,204 \\
\hline Instável & 3 & 7,7 & 9 & 19,1 & 26 & 35,6 & 11,652 & 0,003 \\
\hline Total & 39 & 100 & 47 & 100 & 73 & 100 & & \\
\hline
\end{tabular}

Fonte: elaborado pelas autoras. 
Os dados que se referem à estabilidade das fórmulas cromáticas são apresentados na tabela 2. Em todos os grupos, houve prevalência da fórmula cromática estável. Ainda assim, os resultados evidenciam que houve diferenças estatisticamente significativas entre os três grupos, sendo que a fórmula cromática estável foi significativamente mais frequente nas crianças de 6 anos. A fórmula cromática estável é caracterizada pelo aumento do algarismo CA, isto é, que indica quantas cores foram usadas nas três pirâmides. A fórmula instável também apresentou diferenças estatisticamente significativas entre os grupos, sendo mais frequente nos universitários do que nos demais grupos, conforme teste post hoc.

\section{DISCUSSÃO}

Um primeiro aspecto que chama a atenção é o predomínio da fórmula cromática ampla e redução da fórmula restrita em todas as faixas etárias. Esse dado indica amplo interesse para explorar o material, o que sugere maior empenho voltado a conhecer o ambiente (Villemor-Amaral, 2012). Como a fórmula cromática restrita aponta para características de inibição emocional, costuma ser mais frequente, segundo descrito por Justo e Kolck (1976), em pacientes que apresentam quadros patológicos, tais como depressão, psicose, entre outras. Esse dado é compatível com o esperado para a amostra como um todo, composta por não pacientes.

O aumento da fórmula cromática ampla nas crianças de 6 anos pode ser compreendido com base nas proposiçôes teóricas de Piaget e Wallon, que afirmam que as crianças de 6 anos tendem a demonstrar interesse para o conhecimento e conquista do mundo exterior (Cole \& Cole, 2003; Dantas, 1992). Esse dado corrobora antigas pesquisas sobre o TPC (Bock, 1958; Hartmann, 1959; Schaie, 1964, apud Heiss \& Halder, 1983). Além disso, o aumento de fórmula cromática moderada nos adultos sugere que a gama de escolhas é um pouco menor, sugerindo que esse grupo é um pouco mais seletivo.

No que se refere a estabilidades nas escolhas, chama a atenção o aumento da fórmula cromática estável na idade de 6 anos. É importante considerar que a fórmula cromática do TPC classificada como estável nem sempre deve ser considerada como sinal de maturidade emocional, pois esse indicador, associado a grande amplitude de cores (conforme a tabela 1), costuma resultar num aspecto formal chamado tapete. Os tapetes são caracterizados pelo uso indiscriminado de grande quantidade de cores na pirâmide, indicando menor organização emocional ou cognitiva, conforme descrita por Villemor-Amaral (2014). Esses dados também foram observados no estudo de Villemor-Amaral, Pardini, Tavella \& e Migoranci (2012). 
A fórmula cromática instável, que apareceu aumentada do grupo de adultos universitários $\left(x^{2}=11,652, p=0,003\right)$, indica inconstância nas escolhas (VillemorAmaral, 2012) ou simplesmente o interesse em explorar de diferentes modos o material disponível. Embora esse resultado aparentemente seja contraditório ao que se esperaria na população adulta, é preciso destacar que se trata de adultos universitários e, para Santos e Suehiro (2007), o período de ingresso no curso universitário pode potencializar conflitos por representar a passagem para a vida adulta, não estando essa totalmente consolidada. Esse, portanto, pode ser um sinalizador para a presença desses conflitos.

Verificou-se que a fórmula cromática identificou alguns aspectos típicos das diferentes fases do desenvolvimento, corroborando achados de estudos mais antigos (Villemor-Amaral, 1978). Assim, os dados ora apresentados sugerem que o TPC é um instrumento que pode auxiliar na compreensão do funcionamento psíquico de pessoas com diferentes faixas etárias, possibilitando compreender as variadas mudanças do desenvolvimento humano mencionadas por PerezRamos (2000) e Dantas (1992). Esses dados contribuem para evidências de validade do TPC e corroboram outros estudos sobre esse instrumento de avaliação psicológica, conforme foi apontado na revisão de Silva e Cardoso (2012). Pesquisas que visam à qualificação dos instrumentos são importantes para assegurar que os profissionais possam contar com ferramentas adequadas para uso, conforme apontado por Primi (2010).

\section{CONSIDERAÇÕES FINAIS}

Este estudo foi importante por colocar em debate o indicador fórmula cromática que até então não vinha sendo explorado em pesquisas recentes com o TPC. A fórmula cromática identificou algumas características esperadas para as etapas do desenvolvimento focalizadas nesse trabalho e possibilitou algumas reflexões sobre algumas peculiaridades dos jovens adultos que participaram da pesquisa. Nesse sentido, um dado importante é que todos os integrantes do grupo de adultos eram universitários. Existe a possibilidade de que essa especificidade faça com que o grupo apresente particularidades não necessariamente observadas em adultos não universitários. Assim, considera-se muito importante que essa variável possa ser controlada em estudos futuros.

Mas o estudo permitiu constatar, uma vez mais, que alguns indicadores do Pfister, nesse caso a fórmula cromática, possibilitam discriminar níveis de maturidade emocional de modo compatível com o proposto teoricamente. Entretanto cumpre destacar que uma compreensão da dinâmica emocional da pessoa avaliada requer a integração dos diversos indicadores que aparecem em 
um teste assim como é necessário que sejam consideradas as demais estratégias de avaliação e fontes de informação sobre a pessoa avaliada.

Destaca-se finalmente a grande importância do contínuo estudo em busca de mais evidências de validade, seja do indicador fórmula cromática, seja dos demais componentes da análise e interpretação do TPC. Sem dúvida, avanços com o Pfister repercutem também positivamente nos avanços dos procedimentos de avaliação psicológica como um todo, melhor qualificando a área. 


\section{REFERÊNCIAS}

Adib, S. A. (2008). Evidências de validade do teste de Pfister para indicadores de sintomas de depressão em adolescentes. (Dissertação de Mestrado). Universidade São Francisco, Programa de Pós-Graduação Stricto Sensu em Psicologia, Itatiba.

Bastos-Formighieri, M. S. \& Pasian, S. P. (2012). O teste de Pfister com idosos. Avaliação Psicológica, 11(3), 435-448.

Cardoso, L. M. \& Capitão, C. G. (2007). Avaliação psicológica de crianças surdas pelo Teste das Pirâmides Coloridas de Pfister. Psico-USF, 12(2), 135144.

Carnio, E. C. \& Loureiro, S. R. (1993). Caracterização da percepção do real de pacientes esquizofrênicos, avaliados através das técnicas das pirâmides coloridas de Pfister. Psico, Porto Alegre, 24(1), 35-47.

Cole, M. \& Cole, S. R. (2003). O desenvolvimento da criança e do adolescente. Porto Alegre: Artmed.

Conselho Federal de Psicologia (2003). Resolução n. ${ }^{\circ}$ 2, de 24 de março de 2003. Define e regulamenta o uso, a elaboração e a comercialização de testes psicológicos e revoga a Resolução CFP n. ${ }^{\circ}$ 025/2001. Brasília: CFP, 2003.

Dancey, C. P. \& Reidy, J. (2006). Estatística sem matemática para psicólogos. V. Lorí (Trad.). Porto Alegre: Artmed.

Dantas, H. (1992). A afetividade e a construção do sujeito na psicogenética de Wallon. In Y. De La Taille, M. K. Oliveira \& H. Dantas (1992). Piaget, Vigotsky e Wallon: teorias psicogenéticas em discussão. (pp. 85-100). São Paulo: Summus.

Erikson, E. (1976). Identidade, juventude e crise. Rio de Janeiro: Zahar.

Farah, F. H. Z. (2010). Evidências de validade e precisão no teste de Pfister para crianças de 6 a 10 anos. (Tese de Doutorado). Universidade São Francisco, Programa de Pós-Graduação Stricto Sensu em Psicologia, Itatiba.

Heiss, R. \& Halder, P. (1983). O teste das pirâmides de cores. São Paulo: Vetor.

Justo, H. \& Kolck, T. van (1976). O teste das pirâmides de cores. São Paulo: Vetor. 
Oliveira, E. A., Pasian, S. R. \& Jacquemin, A. (2001). A vivência afetiva em idosos. Psicologia Ciência e Profissão, 21(1), 68-83.

Pasian, S. P., Barroso, J. B. \& Theodozio, D. R. (2014). Estudo normativo com adolescentes de 12 a 14 anos. In A. E. Villemor-Amaral. Teste das pirâmides coloridas de Pfister: versão para crianças e adolescentes. (pp. 107-126). São Paulo: Casa do Psicólogo.

Pérez-Ramos, A. L. M. Q. (2000). Avaliação prospectiva: o exame precoce da criança. In J. A. Cunha. Psicodiagnóstico-V. (pp. 151,157). Porto Alegre: Artes Médicas.

Primi, R. (2010). Avaliação psicológica no Brasil: fundamentos, situação atual e direçóes para o futuro. Psicologia: Teoria e Pesquisa, 26(n. esp.), 25-35.

Resende, A. C. \& Chagas, L. E. (2014). A inteligência geral e o desempenho nas pirâmides coloridas de Pfister em crianças: estudo de validade. In A. E. Villemor-Amaral, Teste das Pirâmides Coloridas de Pfister: versão para crianças e adolescentes. (pp. 147-158). São Paulo: Casa do Psicólogo.

Santos, A. A. A. dos \& Suehiro, A. C. C. (2007). Instrumentos de avaliação da integração e da satisfação acadêmica: estudo de validade. Revista GalegoPortuguesa de Psicoloxía e Educación, 14(1), 7, 107-119.

Silva, L. M. \& Cardoso, L. M. (2012). Revisão de pesquisas brasileiras sobre o teste de Pfister. Avaliação Psicológica, 11(3) (n. esp.), 449-460.

Villemor-Amaral, A. E. (2012). O teste das pirâmides coloridas de Pfister. São Paulo: Casa do Psicólogo.

Villemor-Amaral, A. E. (2014). O teste das pirâmides coloridas de Pfister: versão para crianças e adolescentes. São Paulo: Casa do Psicólogo.

Villemor-Amaral, A. E., Cardoso, L. M., Pavan, P. M P., Tavella, R. R. \& Biasi, F. C. (2014a). Estudos recentes de validade e precisão. In A. E. Villemor-Amaral, Teste das Pirâmides Coloridas de Pfister: versão para crianças e adolescentes. (pp. 127-146). São Paulo: Casa do Psicólogo.

Villemor-Amaral, A. E., Cardoso, L. M., Pavan, P. M P., Tavella, R. R., Biasi, F. C. \& Miguel, F. K. (2014b). Estudo normativo com crianças de 6 a 12 anos. In A. E. Villemor-Amaral, Teste das pirâmides coloridas de Pfister: versão para crianças e adolescentes. (pp. 55-106). São Paulo: Casa do Psicólogo. 
Villemor-Amaral, A. E., Farah, F. H. Z. \& Primi, R. (2004). O teste das pirâmides coloridas e o transtorno do pânico. Psicologia Estudos, 9(2), 301-307.

Villemor-Amaral. A. E., Miguel, F. K., Biasi, F., Migoranci, P. B., Pavan, P. M. P. \& Tavella, R. R. (2012, maio). O teste das pirâmides coloridas de Pfister: um estudo de fidedignidade (pôster). $18^{\circ}$ Encontro de Iniciação Científica, $11^{\circ}$ Encontro de graduação, 7o Encontro de Extensão Universitária, Seminários de Estudo do Homem Contemporâneo - V Ciclo. Bragança Paulista: Universidade São Francisco.

Villemor-Amaral, A. E., Pardini, P. M., Tavella, R. R., Biasi, F. C. \& Migoranci, P. B. (2012). Evidências de validade do teste de Pfister para avaliação de crianças. Avaliação Psicológica, 11(3) (n. esp.), 423-434.

Villemor-Amaral, A. E., Pianowski, G. \& Gonçalves, C. M. T. S. (2008). Estudo normativo com o Pfister: uma amostra da região nordeste brasileira. Avaliação Psicológica, 7(2), 181-188.

Villemor-Amaral, A. E., Primi, R., Farah, F. H. Z., Cardoso, L. M. \& Franco, R. R. C. (2003). Revisão das expectativas no Pfister para uma amostra normativa. Avaliação Psicológica, 2(2), 185-188.

Villemor-Amaral, A. E., Primi, R., Farah, F. H. Z., Silva, S. M., Cardoso, L. M. \& Franco, R. R. C. (2004). A depressão no teste das pirâmides coloridas de Pfister. Paidéia: Cadernos de psicologia e educação, 14 (28), 169-179.

Villemor-Amaral, A. E., Primi, R., Franco, R. R. C., Farah, F. H. Z, Cardoso, L. M. \& Silva, T. C. (2005). O teste de Pfister e sua contribuição para o diagnóstico da esquizofrenia. Revista do Departamento de Psicologia, Universidade Federal Fluminense, 17(2), 89-98.

Villemor-Amaral, A. E., Silva, T. C. \& Primi, R. (2002). O teste das pirâmides coloridas de Pfister e o transtorno obsessivo compulsivo. Avaliação Psicológica, 1(2), 133-139.

Villemor-Amaral, A. E., Silva, T. C. \& Primi, R. (2003). Indicadores de alcoolismo no teste das pirâmides coloridas de Pfister. Psico - USF, 8(1), 33-38.

Villemor Amaral, F. (1978). Pirâmides coloridas de Pfister. Rio de Janeiro: Cepa. 